\title{
Rosazea - das Erbe keltischer Ahnen?
}

\author{
Es ist womöglich nur eine kleine "Macke“ im Erbgut von Rosazea-Patienten, die dazu führt, dass \\ sie überempfindlich auf Sonnenlicht, Hitze oder Kälte reagieren. Für ihre Vorfahren, die Kelten, \\ stellte diese Mutation vermutlich einen Überlebensvorteil dar. Im Gespräch mit der ästhetischen \\ dermatologie \& kosmetologie erklärt Prof. Bodo Melnik, wie Vitamin-D-Mangel bei den \\ keltischen Ahnen mit dem übermäßigen Vorkommen des pathogenetisch relevanten Peptids \\ Cathelicidin in der Haut heutiger Rosazea-Patienten zusammenhängt.
}

\begin{abstract}
? Herr Professor Melnik, worin besteht der evolutionsbedingte „Segen" für die Kelten, den Sie im Titel ihrer Arbeit propagieren? Melnik: Ursprünglich hatten die Kelten ein Problem: Sie lebten in sonnenarmen Regionen - waren also Nordlichter. In den Wintermonaten war es ihnen aufgrund von Vitamin-D-Mangel nicht möglich, ihre Infektabwehr optimal zu aktivieren. Infolgedessen waren sie anfälliger, z. B. für Tuberkulose, aber auch für Virusund Pilzinfektionen, bei deren Abwehr das antimikrobielle Peptid Cathelicidin eine zentrale Rolle spielt. Weil die Vitamin-Dinduzierte Cathelicidin-Bildung geografisch bedingt vor allem in den Wintermonaten nur unzureichend funktionierte, hatten die Kelten ein erhöhtes Risiko, an Infekten zu sterben.
\end{abstract}

? Worin liegt nun der Vorteil und was hat das mit den RosazeaPatienten unserer Zeit zu tun?

Melnik: Die Cathelicidin-Transkription wird nicht nur durch Vitamin D aktiviert, sondern auch Vitamin-D-unabhängig durch einen zweiten Signalweg. Dieser Weg wird durch sogenannten ER-Stress der Zelle (s. Kasten) aktiviert und führt zur Aktivierung des Transkriptionsfaktors C/EBPa. Ich habe postuliert, dass Rosazea-Patienten infolge einer noch unbekannten Mutation ihre ER-Stress-Empfindlichkeit „hochgetunt“ haben, um über diesen alternativen Signalweg im Winter ausreichend Cathelicidin für die Infektabwehr zur Verfügung zu haben.

? Wie lassen sich in Ihrem Konzept die bekannten Rosazea-Trigger erklären?

Melnik: Alle bekannten Rosazea-Trigger wie UV-Licht, Hitze, Kälte und scharfe Speisen lassen sich als Stressoren des ER erklären. Sie überhöhen damit die Cathelicidin-Bildung in der Haut von Rosazea-Patienten, womit eine Cathelicidin-vermittelte Entzündung provoziert wird.

? Was passiert, wenn man Rosazea-Patienten Vitamin D gibt? Könnte man damit bewirken, dass der intrinsische, Vitamin-D-unabhängige Pfad nicht so stark aktiviert wird?

Melnik: Nein, im Gegenteil, das wäre ein Eigentor! Beide Aktivierungswege führen unabhängig voneinander zur Cathelicidin-Expression und damit zu einer Exazerbation des entzündlichen Geschehens.

? Also auf keinen Fall Vitamin D geben?

Melnik: Nein! Das ist ja auch unsere klinische Erfahrung: Bei Rosazea-Patienten, die gleichzeitig an Psoriasis leiden, beobachten wir häufig ein Aufflammen der Rosazea bei topischer Anwendung des Vitamin-D-Analogons Calcipotriol im Gesicht.

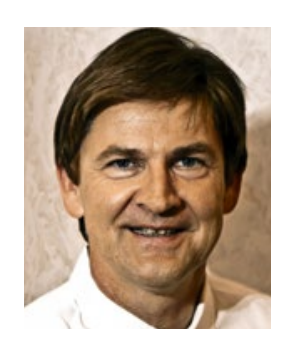

Prof. Dr. Bodo C. Melnik

Dermatologie, Umweltmedizin und

Gesundheitstheorie,

Universität Osnabrück

? Stichwort Arzneimitteltherapie: Sie schreiben, dass die Medikamente, die bei Rosazea wirksam sind, in die genannte Signalkaskade eingreifen. Ist das für Sie der Beweis, dass Ihre Theorie stimmt?

Melnik: Ein indirekter Beweis. Nehmen wir z. B. Isotretinoin: Bei Rosazea ist der Toll-like Rezeptor 2 aktiviert. Dieser lässt sich mit Isotretinoin wieder herunter regulieren. Außerdem reduziert Isotretinoin die Cathelicidinbildung der Sebozyten. Neu eingeführt bei Rosazea wurde Ivermectin: Dieses hemmt ein kritisches Enzym, die p38-Kinase, durch deren Mitwirkung der Transkriptionsfaktor C/EBPa aktiviert wird. Metronidazol, Tetrazykline und Azelainsäure schließlich hemmen das Enzym Kallikrein, das die proteolytische Spaltung von Cathelicidin zum aktiven Peptid LL-37 katalysiert. All diese Substanzen hat man als Zufallsbefunde empirisch bei Rosazea eingesetzt. Und jetzt passen all diese unterschiedlichen Pharmazeutika in eine Signalkaskade. Ich würde sagen, das kann kein Zufall sein.

? Worauf könnte sich die zukünftige Arzneimittelforschung bei Rosazea konzentrieren?

Melnik: Wenn wir den Gendefekt in der Regulation des ERStresses bei Rosazea lokalisiert haben, müsste es gelingen, die abgesenkte ER-Stress-Schwelle wieder zu normalisieren. Derzeit gibt es bereits Aktivitäten an der Universität San Francisco, die sich auf den Sphingosin-Stoffwechsel konzentrieren. Interessanterweise besteht eine erhöhte Assoziation von Rosazea mit multipler Sklerose (MS). Auch bei MS wird überhöhter ERStress als pathogenetischer Mechanismus diskutiert und Rezeptorantagonisten von Sphingosin-1-Phosphat klinisch erprobt. Derselbe ER-Stress-Signalweg, der bei den Kelten die Infektanfälligkeit gebessert hat, scheint möglicherweise die Disposition für MS zu erhöhen.

Das Interview führte Dr. Elke Oberhofer. 